Bull. Austral. Math. Soc.

$60 \mathrm{G} 17,60 \mathrm{G} 51,26 \mathrm{~A} 33$

VoL. 67 (2003) [523-526]

\title{
Stochastic Analysis and Approximation of Fractional Diffusion
}

\author{
R. MCVINISH
}

In the Eulerian theory of turbulent diffusion, the concentration field is commonly assumed to satisfy the advection-diffusion equation. On the other hand, in the Lagrangian theory, this is equivalent to assuming the particle trajectories satisfy an Itô stochastic differential equation. In certain applications, such as diffusion in porous media with fractal geometry, the data available indicates that the behaviour of the particles is very different and the resulting process is referred to as fractional diffusion. Specifically, in this thesis fractional diffusion is characterised by particle trajectories possessing one or more of the following properties: (i) long-range dependence, (ii) heavy tails and non-Gaussian behaviour and (iii) multifractal sample paths. It is the purpose of this thesis to present some new results on fractional diffusion in the areas of stochastic analysis and numerical approximation.

Chapter one of the thesis reviews the relevant literature on fractional diffusion. Emphasis is placed on Lévy motion, long range dependence and their connection to the fractional calculus.

Chapter two considers fractional Riesz-Bessel motion and related processes. In that Chapter it is seen that fractional Riesz-Bessel motion can arise as a limit of a normalised convolution of a long-range dependent process with the Bessel potential. The results of this chapter have been published in Anh et al. [2].

Chapter three studies the path behaviour and approximation of the class of linear fractional differential equations driven by Lévy noise introduced in Anh et al. [1]. A special case of this class is the Stokes-Boussinesq-Langevin equation that describes the motion of a spherical particle in a viscous fluid under thermal noise. An important result of this chapter is the refinement of a result by Hardy and Littlewood [4] regarding the mapping property of fractional integrals on the space of Hölder continuous functions. This result allows conditions to be determined so that the solution process has a semimartingale representation and for the multifractal spectrum to be determined by the driving noise. The numerical approximation of the solution was based on the Green function being completely monotonic for a range of its parameters. This allowed the solution to

Received February 25, 2003

Thesis submitted to Queensland University of Technology, March 2002. Degree approved September 2002. Supervisors: Associate Professor Vo Anh and Dr Rodney Wolff.

Copyright Clearance Centre, Inc. Serial-fee code: 0004-9727/03 \$A2.00+0.00. 
be written as the limit of a system of integer order differential equations with Lévy noise which could then be approximated in a standard way. Treating the approximation as a pathwise integral and applying the Love-Young inequality to the error proved uniform convergence. A related model that is more suitable for non-stationary processes with stationary increments is studied in Chapter four.

The previous chapters of the thesis have essentially looked at the Lagrangian approach to fractional diffusion. The only case where there is a clear connection between Lagrangian and Eulerian approaches in fractional diffusion is in the description of Lévy motion. One of the better known examples of a non-Gaussian Lévy motion is $\alpha$-stable motion. Feller [3] was able to generate every stable density as the solution to a fractionalin-space diffusion equation where the spatial operator was a generalisation of the inverse of the Riesz potential. In this chapter a fractional-in-space diffusion is considered where the spatial operator is obtained from the composition of the inverses of the Riesz and Bessel potentials. It is proved that, under certain conditions on the parameters, this spatial operator is the infinitesimal generator of a Lévy semigroup. The Lévy motion behaviour varies considerably with the parameters, from a compound Poisson process to a process with an infinite number of jumps in finite time to the sum of a Brownian motion and a compound Poisson process. More generally, it was proved that the operator is an infinitesimal generator of a strongly continuous holomorphic semigroup of angle $\pi / 2$ for a wide range of its parameters. The varying behaviour displayed by the Lévy motion can also be related to the infinitesimal generator for this range of parameters. Numerical approximation of the fractional diffusion equation was discussed, together with applications to certain stochastic evolution equations.

At present, the theory of fractional diffusion is considerably weaker than that of the classical diffusion case. An important step in developing a more complete theory is the development of fractional stochastic differential equations that can include nonlinear behaviour. In Chapter six, the existence and uniqueness of the solution is proved under certain conditions. A splitting-type method for the numerical approximation of the solution to some simple cases is presented together with the proof of convergence. Chapter seven briefly summarises the main results of the thesis and indicates avenues for further research.

\section{REFERENCES}

[1] V.V. Anh, C.C. Heyde and N.N. Leonenko, 'Dynamic models of long-memory processes driven by Lévy noise with applications to finance and macroeconomics', J. Appl. Probab. 39 (2002), 730-747.

[2] V.V. Anh, N.N. Leonenko and R. McVinish, 'Models for fractional Riesz-Bessel motion and related processes', Fractals 9 (2001), 329-346.

[3] W. Feller, 'On a generalization of Marcel Riesz' potential and the semi-groups generated by them', in Comm. Sém. Matém. Université de Lund, 1952, pp. 73-81. 
[4] G.H. Hardy and J.E. Littlewood, 'Some properties of fractional integrals', Proc. London Math. Soc. (2) 24 (1925), 37-41.

School of Mathematicsl Sciences

Queensland University of Technology

GPO Box 2434

Brisbane QLD 4001

Australia 